Article

\title{
Analysis of Intestinal Mucosa Integrity and GLP-2 Gene Functions upon Porcine Epidemic Diarrhea Virus Infection in Pigs
}

\author{
Yajing Zhou ${ }^{1}$, Zhanshi Ren ${ }^{1}$, Shuai Zhang ${ }^{1}$, Haifei Wang ${ }^{1}$, Shenglong Wu ${ }^{1,2}$ and Wenbin Bao ${ }^{1,2, *(D)}$ \\ 1 Key Laboratory for Animal Genetics, Breeding, Reproduction and Molecular Design, College of Animal \\ Science and Technology, Yangzhou University, Yangzhou 225009, China; xxxcandygd@163.com (Y.Z.); \\ Renzhanshi0422@163.com (Z.R.); shuai_zhang1990@163.com (S.Z.); wanghaiffei@126.com (H.W.); \\ slwu@yzu.edu.cn (S.W.) \\ 2 Joint International Research Laboratory of Agriculture \& Agri-Product Safety, The Ministry of Education \\ of China, Yangzhou University, Yangzhou 225009, China \\ * Correspondence: wbbao@yzu.edu.cn
}

check for updates

Citation: Zhou, Y.; Ren, Z.; Zhang, S.; Wang, H.; Wu, S.; Bao, W. Analysis of Intestinal Mucosa Integrity and GLP-2 Gene Functions upon Porcine Epidemic Diarrhea Virus Infection in Pigs. Animals 2021, 11, 644. https:// doi.org/10.3390/ani11030644

Academic Editor: Paolo Bosi

Received: 3 February 2021

Accepted: 24 February 2021

Published: 1 March 2021

Publisher's Note: MDPI stays neutral with regard to jurisdictional claims in published maps and institutional affiliations.

Copyright: (c) 2021 by the authors. Licensee MDPI, Basel, Switzerland. This article is an open access article distributed under the terms and conditions of the Creative Commons Attribution (CC BY) license (https:// creativecommons.org/licenses/by/ $4.0 /)$.
Simple Summary: Porcine epidemic diarrhea virus causes serious diarrhea in suckling piglets, resulting in huge economic losses in the pig industry. A better understanding of porcine epidemic diarrhea virus (PEDV) pathogenesis and identification of the responsive genes will contribute to controlling PEDV infection. Therefore, screening and identifying PEDV-resistance functional genes and then implementing disease resistance breeding have important scientific significance. This study explores the regulatory roles of the GLP-2 gene in regulating PEDV infection and in repairing the intestinal mucosa damage associated with PEDV infection. The findings may contribute to the identification of resistant genes or genetic markers for disease resistance breeding in pigs.

Abstract: Porcine epidemic diarrhea virus (PEDV) infects intestinal epithelial cells, destroys the intestinal mucosal barrier and then causes diarrhea in piglets. Glucagon-like peptide-2 (GLP-2) is a specific intestinal growth hormone that promotes the repair of damaged intestinal mucosa and improves the intestinal barrier. In this study, we investigated the functions of porcine GLP-2 gene in regulating PEDV infection. The intestinal tissues with damaged intestinal structures caused by PEDV infection were first confirmed and collected. Expression analysis indicated that the GLP-2 gene was expressed in the duodenum, jejunum and ileum tissues, and the mRNA level was significantly down-regulated in jejunum and ileum of piglets with damaged intestinal mucosa. Infection of PEDV to porcine small intestinal epithelial cells in vitro showed that GLP-2 gene was significantly decreased, which was consistent with the expression pattern in intestinal tissues. In addition, we silenced the GLP-2 gene by shRNA interfering and found that the copy numbers of PEDV were remarkably increased in the GLP-2 gene silencing cells. Our findings suggest that the GLP-2 gene was potentially involved in regulating PEDV infection and in maintaining the integrity of the intestinal mucosal barrier structure, which could contribute to our understanding of the mechanisms of PEDV pathogenesis and provide a theoretical basis for the identification and application of resistant genes in pig selective breeding for porcine epidemic diarrhea.

Keywords: pigs; PEDV; GLP-2 gene; intestinal mucosa barrier; gene expression

\section{Introduction}

Porcine epidemic diarrhea is a contagious disease caused by porcine epidemic diarrhea virus (PEDV), with clinical manifestations including severe diarrhea, vomiting, anorexia and dehydration. PEDV can infect pigs at all stages of growth and result in high mortality for suckling piglets, causing huge economic losses to the pig industry. PEDV, an invasive single-stranded positive-stranded RNA virus, belongs to the genus Alphacoronavirus in 
the family Coronaviridae [1]. The virus genome is about $28 \mathrm{~kb}$ in length, encoding two polyproteins (pp1a and pp1b), an accessory protein (open reading frame 3, ORF3) and four structural proteins (spike, S; envelope, E; membrane, $\mathrm{M}$; and nucleocapsid, N) [2,3]. ORF3 protein causes the release of the virus by forming ion channels in the host [4]; $S$ protein binds to the host surface receptor to regulate the replication of PEDV [5]; M protein interacts with $\mathrm{S}$ and $\mathrm{N}$ proteins, which plays an important role in the process of virus budding, assembly and replication [6,7]. PEDV mainly infects the intestinal villi cells and mesenteric lymph nodes of the pig small intestine. It destroys the structure of the intestine, damages the intestinal epithelial cells, causes nutrient absorption disorders and then causes diarrhea [8].

The intestine is an important part of the systemic immune system [9]. The intestinal mucosal barrier is a complex defense system composed of intestinal mucosal epithelial cells, intestinal mucosal immune system and intestinal microbes. The intestinal mucosal barrier protects the body by preventing pathogens, antigens and bacterial toxins from invading the mucosa and preventing the absorption of toxins [10]. It has been reported that as the most important component of the intestinal mucosal barrier, the intestinal mucosal epithelial cells play a regulatory role through tight junction proteins and mucins and are the first line of defense against gastrointestinal infections [11]. Tight junctions are an important connection method of the intestinal epithelium. The impaired intestinal barrier function of pigs is often related to the impaired tight junctions $[12,13]$. The intestinal mucosal immune system resists the invasion of pathogenic microorganisms through immunosuppression and protects important physiological functions of the body [14]. PEDV mainly invades the body through intestinal epithelial cells; therefore, intestinal mucosal epithelial cells and the intestinal mucosal immune system play an important role in defense against PEDV infection.

Glucagon-like peptide-2 (GLP-2) is a 33-amino-acid peptide, which is mainly composed of the post-translational processing of the glucagon pro-chain in the small intestinal endocrine L cells. It was produced in the small intestine along with GLP-1. The release of GLP-2 is mainly caused by direct contact with long-chain fatty acids in the terminal ileum. Studies have shown that GLP-2 is mediated by a specific G protein-coupled receptor, which is only found in the surrounding tissues of the intestine, such as L cells adjacent to intestinal crypts, enteric neuron and scattered populations of myofibroblasts [15-17]. As a specific intestinal growth hormone, GLP-2 inhibits cell apoptosis by promoting the proliferation and differentiation of intestinal cells [18]. It can also reduce the permeability of intestinal epithelial cells, promote the repair of damaged intestinal mucosa and improve the intestine tract barrier function $[19,20]$. Moran et al. [21] found that GLP-2 can promote the repair of intestinal mucosa by promoting the expression of tight junction proteins Zona Occluden-1 (ZO-1), Occludin and Claudine-1.

PEDV invades the body mainly through intestinal epithelial cells. The GLP-2 gene plays a biological function in intestinal mucosal epithelial cells and the intestinal mucosal immune system. However, little is known about the regulatory effects of GLP-2 gene on PEDV-induced intestinal injury. The aim of this study was to explore the effects of GLP-2 gene expression on intestinal mucosal integrity and on the regulation of PEDV infections. Our findings could provide a theoretical basis for further research on the role and molecular mechanism of GLP-2 gene in resisting porcine epidemic diarrhea.

\section{Materials and Methods}

\subsection{Ethics Statement}

The animal study proposal was approved by the Institutional Animal Care and Use Committee (IACUC) of the Yangzhou University Animal Experiments Ethics Committee (permit number: SYXK (Su) IACUC 2012-0029). All experimental methods were conducted in accordance with the related guidelines and regulations. 


\subsection{Experimental Animal}

The experimental pigs were selected from a large-scale pig farm in Jiangsu Province. Six 7-day-old ternary hybrid piglets that were confirmed to be infected with PEDV by qPCR and six healthy piglets with the same birth weight through half-sib selection were used [22]. Two groups of piglets were slaughtered, and then tissue samples of duodenum, jejunum and ileum were collected, stored in liquid nitrogen on-site and transferred to $-70{ }^{\circ} \mathrm{C}$ refrigerator for storage for later use.

\subsection{Paraffin Section Preparation and Hematoxylin and Eosin (HEE) Staining}

We separated the middle part of the duodenum, the jejunum and the ileum by about $3 \mathrm{~cm}$. Then these tissue samples were rinsed with PBS and fixed in $4 \%$ paraformaldehyde solution for $24 \mathrm{~h}$. They were sliced after dehydration, transparency, wax immersion and embedding. They were put in $45^{\circ} \mathrm{C}$ and then took out the slices and baked slices at $60{ }^{\circ} \mathrm{C}$ for $1 \mathrm{~h}$. After H\&E staining, the morphology of each intestine segment was observed with a microscope (Leica, Heidelberg, Germany).

\subsection{Primer Design and Synthesis}

The porcine GLP-2 (NM_214324.1) gene and PEDV M (AF017079.1) gene sequences published in the GenBank database, Premier 5.0 software, was used to design qPCR primers, with GAPDH and ACTB genes as internal control. Primers were synthesized by Sangon Biotech Co., Ltd. (Shanghai, China). Primer information is shown in Table 1.

Table 1. The primer sequence of genes for qPCR.

\begin{tabular}{ccc}
\hline Gene & \multicolumn{1}{c}{ Primer Sequence } & Product Length (bp) \\
\hline \multirow{2}{*}{ GLP-2 } & F: 5'-ACTCACAGGGCACGTTTACCA-3' & 150 \\
& R: 5'-AGGTCCCTTCAGCATGTCTCT-3' & \\
PEDVM & F: 5'-AGGTCTGCATTCCAGTGCTT-3' & 216 \\
& R: 5'-CCTGCCCAGATTCAGCAAAG-3' & \multirow{2}{*}{187} \\
GAPDH & F: 5'-ACATCATCCCTGCTTCTACTGG-3' & \\
& R: 5'-CTCGGACGCCTGCTTCAC-3' & 149 \\
$\beta$-ACTIN & F: $5^{\prime}$-TGGCGCCCAGCACGATGAAG-3' & \\
& R: 5'-GATGGAGGGGCCGGACTCGT-3' & \\
\hline
\end{tabular}

\subsection{Construction of shRNA Silencing Cells}

The CDS region sequence of pig GLP-2 (NM_214324.1) gene was obtained from the gene sequence published in the GenBank database. Three target sequences (shRNA1, shRNA2, shRNA3) through the shRNA target gene design website (http:/ / rnaidesigner. thermofisher.com/rnaiexpress/, 15 July 2020) were screened. Primers were synthesized by Sangon Biotech Co., Ltd. (Shanghai, China). Detailed information is shown in Table 2. The three pairs of shRNA sequences were oligo annealed and connected. The oligo annealing procedure and conditions were as follows: $5 \mu \mathrm{L}$ each for positive and negative strands, $2 \mu \mathrm{L}$ annealing buffer $(10 \times), 8 \mu \mathrm{L} \mathrm{ddH_{2 }} \mathrm{O}$ and then $95^{\circ} \mathrm{C} 5 \mathrm{~min}$, natural cooling at room temperature for $20 \mathrm{~min}$. The connection procedure and conditions were as follows: $4 \mu \mathrm{L}$ of oligo product, $2 \mu \mathrm{L}$ of linearization vector, $1 \mu \mathrm{L}$ of T4 DNA ligase, $4 \mu \mathrm{L}$ of ligation buffer $(5 \times), 9 \mu \mathrm{L}$ of $\mathrm{ddH}_{2} \mathrm{O}$ and then $16{ }^{\circ} \mathrm{C}$ overnight connection. After transforming the vector into $\mathrm{DH} 5 \alpha$, we performed plasmid extraction. According to the instruction of lipofectamine 2000 (Invitrogen, Grand Island, NY, USA) transfection reagent, we transfected the transfection reagent, plasmid and Opti-MEM (Gibco, Grand Island, NY, USA) into the 12-well plate. After $48 \mathrm{~h}$ of drug screening, we observed the fluorescence. When the cell confluency was greater than $80 \%$, the cells were collected and total RNA was extracted to verify the interference efficiency. 
Table 2. The oligomeric single-stranded DNA sequence of shRNAs.

\begin{tabular}{|c|c|}
\hline Name & Sequence of Oligo \\
\hline \multirow{2}{*}{ shRNA1 } & F: 5'-CACCGCCCTCTCGATGATCCAGATTTCAAGAGAATCTGGATCATCGAGAGGGTTTTTTG -3' \\
\hline & 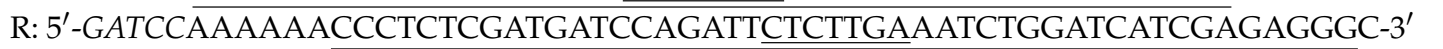 \\
\hline \multirow{2}{*}{ shRNA2 } & 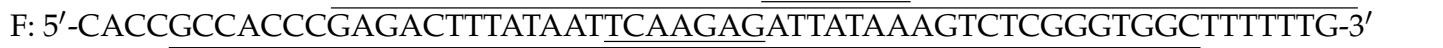 \\
\hline & R: 5'-GATCCAAAAAAGCCACCCGAGACTTTATAATCTCTTGAATTATAAAGTCTCGGGTGGC-3' \\
\hline \multirow{2}{*}{ shRNA3 } & F: 5'-CACCGCTTTGTGGCTGGATTGTTTCAAGAGAACAATCCAGCCACAAAGTTTTTTG-3' \\
\hline & R: 5'-GATCCAAAAAACTTTGTGGCTGGATTGTTCTCTTGAAACAATCCAGCCACAAAGC-3’ \\
\hline \multirow{2}{*}{ shRNA NC } & F: 5'-CACCGTTCTCCGAACGTGTCACGTCAAGAGATTACGTGACACGTTCGGAGAATTTTTTG-3' \\
\hline & R: 5' - GATCCAAAAAATTCTCCGAACGTGTCACGTAATCTCTTGACGTGACACGTTCGGAGAAC-3' \\
\hline
\end{tabular}

The italic part is the enzyme cutting site; the single-underlined part is the interference sequence and its complementary sequence, and the double-underlined part is the loop sequence.

\subsection{PEDV Infects IPEC-J2 Cells and shRNA Silencing Cells}

The GLP-2 gene interfering IPEC-J2 cells and control IPEC-J2 cells were seeded in 6-well plates at $1 \times 10^{5}$. They were cultured in Dulbecco's modified Eagle medium (DMEM; Gibco, Grand Island, NY, USA) containing 10\% fetal bovine serum (FBS; Gibco, Grand Island, NY, USA) at $5 \% \mathrm{CO}_{2}$ and $37{ }^{\circ} \mathrm{C}$ and then cultured for $12 \mathrm{~h}$. PEDV was added to the experimental wells at $\mathrm{MOI}=0.1$, and cells without virus infection were set as mock cells. After incubation for $48 \mathrm{~h}$, the cells were collected and used for total RNA isolation.

\subsection{Total RNA Extraction and $q P C R$}

The Trizol method was used to extract total RNA from piglets' intestinal tissues and IPEC-J2 cells. The extraction steps were strictly in accordance with the Trizol Reagent (Takara, Beijing, China). The purity of RNA was detected by $2.2 \%$ formaldehyde denaturation agarose gel electrophoresis and NanoDrop ND-1000 micro nucleic acid concentration analyzer. RNA was anti-transcribed into cDNA and used as template. We used qPCR to quantify gene expression, with a volume of $20 \mu \mathrm{L}$ containing cDNA template $2.0 \mu \mathrm{L}$, forward and reserve primers each $0.4 \mu \mathrm{L}(10 \mu \mathrm{mol} / \mathrm{L})$, ROX Reference Dye II $(50 \times) 0.4$ $\mu \mathrm{L}$, SYBR Green qPCR Master Mix $(2 \times) 10 \mu \mathrm{L}$, ddH2O $6.8 \mu \mathrm{L}$. The reaction conditions were as follows: $95^{\circ} \mathrm{C}$ for $5 \mathrm{~min}$, followed by 40 cycles of $95^{\circ} \mathrm{C}$ for $10 \mathrm{~s}$ and $60^{\circ} \mathrm{C}$ for 30 $\mathrm{s}$. We analyzed the melting curve after the amplification was completed and judged the unity of PCR amplification according to whether there was an $85 \pm 0.8^{\circ} \mathrm{C}$ peak on the melting curve.

\subsection{Western Blotting Analysis}

Cells were washed twice with PBS and incubated on ice with RIPA Lysis and Extraction Buffer (biosharp, BL509A, wobio, Nanjing, China) and Protease Inhibitor Cocktail (biosharp, BL612A, wobio, Nanjing, China). The lysates were denatured for $10 \mathrm{~min}$ in $5 \times$ SDS-PAGE loading buffer and separated with SDS-PAGE. The proteins were then transferred to nitrocellulose Western blotting membranes (millipore, IPVH08100, wobio, Nanjing, China). The membranes were blocked with PBS containing 5\% nonfat dry milk and $0.2 \%$ Tween 20 for $2 \mathrm{~h}$ at room temperature. Then, the membranes with the primary antibody were incubated at $4{ }^{\circ} \mathrm{C}$ for the whole night. After the membranes were washed with PBS containing $0.1 \%$ Tween $20(0.1 \%$ PBST), they were incubated with the corresponding secondary antibody (conjugated with HRP) at room temperature for $2 \mathrm{~h}$ and detected with enhanced chemiluminescence (ECL) (Thermo Fisher Scientific, 34580, Waltham, MA, USA).

\subsection{Statistical Analysis}

According to the PEDV amplification standard curve $y=-3.3354 \lg (\mathrm{x})+37.832$ ( $\mathrm{y}$ represents the number of $\mathrm{Ct}$ cycles, $\mathrm{x}$ represents the logarithm of the virus copy number based on the base 10), the quantitative $\mathrm{Ct}$ value of the PEDV M gene was substituted into the formula and then the number of copies of PEDV was calculated. The $2^{-\Delta \Delta \mathrm{Ct}}$ method was used to detect the transcript levels of the genes. $\Delta \Delta \mathrm{Ct}=$ (the average $\mathrm{Ct}$ value of the 
target gene in the test group - the average $\mathrm{Ct}$ value of the internal reference gene in the test group) - (the average Ct value of the target gene in the control group - the average $\mathrm{Ct}$ value of the internal reference gene in the control group). SPSS 21.0 software was utilized to carry out an independent sample t-test, and data are expressed as means \pm standard deviation (Mean $\pm \mathrm{SD}$ ).

\section{Results}

\subsection{Hematoxylin and Eosin (HEE) Staining of the Small Intestine}

In order to detect the difference in intestinal structures between PEDV-infected piglets and healthy piglets, the intestinal mucosa of piglets was stained by H\&E staining. The results showed that the intestinal villi of the diseased piglets were severely damaged, the intestinal mucosal epithelial cells were necrotizing and shedding, the lamina propria were exposed and the intestinal glands were atrophied. In healthy piglets, the structure of the intestinal mucosa was complete, with distinct layers, and the intestinal mucosal epithelial cells had a clear outline and regular arrangement (Figure 1). The above results indicated that the intestines exhibited obvious lesions, and the intestinal mucosa was damaged post-PEDV infection. The intestines derived from PEDV-infected and healthy piglets were used for subsequent experiments

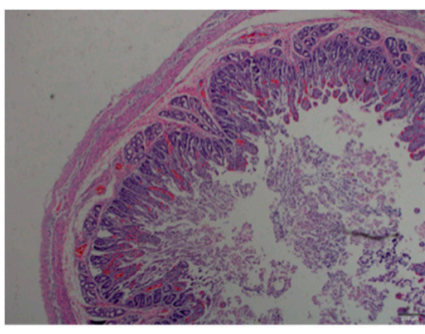

A

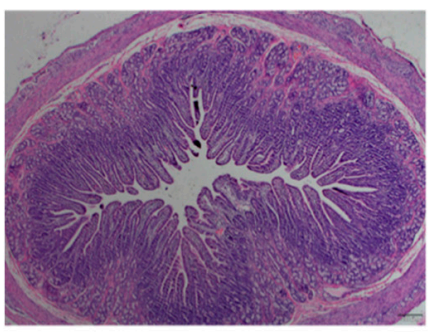

D

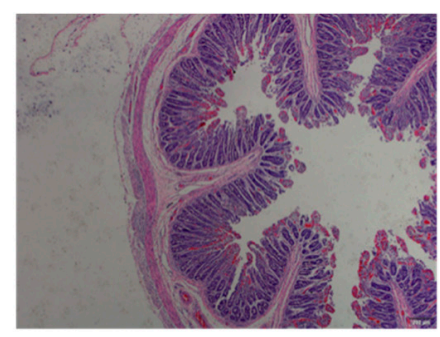

B

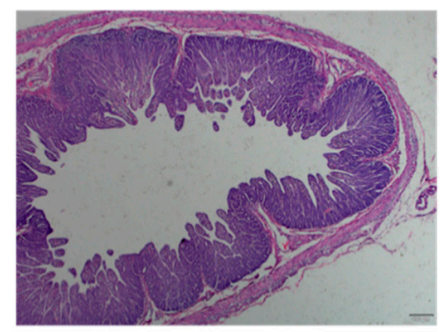

E

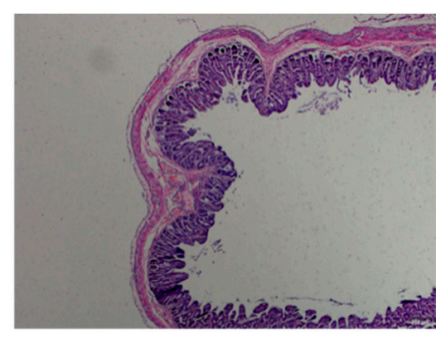

C

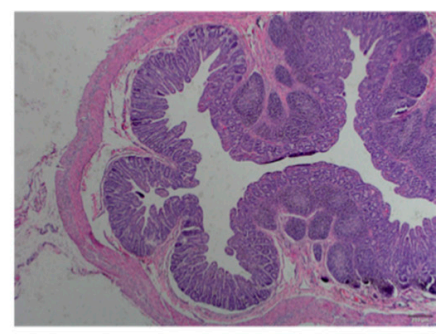

F

Figure 1. H\&E-staining microscopic examination of the small intestine tissue from porcine epidemic diarrhea virus (PEDV)-infected and normal piglets. (A-C) indicate the duodenum, jejunum, ileum tissues of PEDV-infected piglets $(40 \times)$; $($ D-F) indicate the duodenum, jejunum, ileum tissues of normal piglets $(40 \times)$.

\subsection{Total RNA Quality Detection and $q P C R$ Primer Specificity}

The total RNA extracted from the intestinal tissue and IPEC-J2 were subjected to $2.2 \%$ formaldehyde denaturation agarose gel electrophoresis, and the three bands of $28 \mathrm{~S}$, $18 \mathrm{~S}$ and $5 \mathrm{~S}$ were clear (Figure S1). This proved that there was no DNA contamination band and degradation. The A260/A280 of the samples were 1.8-2.0, indicating that the completeness and purity of the RNA extraction were good, and it could be used for subsequent experiments. The qPCR amplification and melting curves for the GLP-2 and PEDV M genes showed that the PCR product had only one definite peak and no primer dimer or non-specific product (Figures S2 and S3) 
3.3. Expression Analysis of GLP-2 Gene in Intestinal Segments of the Healthy and Damaged Intestinal Mucosa Group

Gene expression quantitative results found that the GLP-2 gene was expressed in the duodenum, jejunum and ileum. In addition, the expression level of GLP-2 gene in the jejunum and ileum of the intact intestinal mucosa group was significantly higher than that in the intestinal mucosal damage group $(p<0.01)$ (Figure 2). The results showed that when piglets were infected with PEDV, the expression of the GLP-2 gene in jejunum and ileum of piglets decreased significantly.

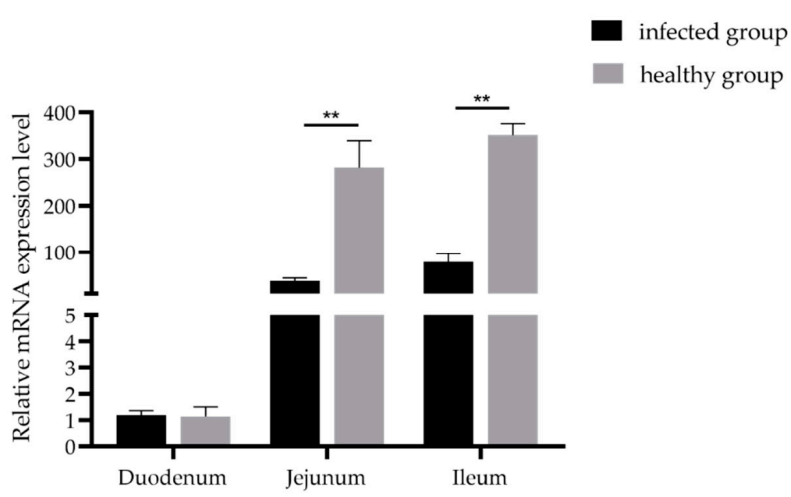

Figure 2. Expression levels of the GLP-2 gene in the PEDV-infected group and healthy group. ** $p<0.01$. Bars represent the mean $\pm \mathrm{SD}(n=3)$.

\subsection{Analysis of Differential Expression of GLP-2 Gene in IPEC-J2 Cells with PEDV Infection}

To further explore the relationship between GLP-2 gene expression and PEDV infection, we used qPCR to detect the difference between the expression level of GLP-2 gene in IPEC$\mathrm{J} 2$ cells infected with PEDV for $48 \mathrm{~h}$ and uninfected control cells. The results showed that the expression level of GLP-2 gene in PEDV-infected cells was significantly lower than that in the control group $(p<0.01)$ (Figure 3$)$.

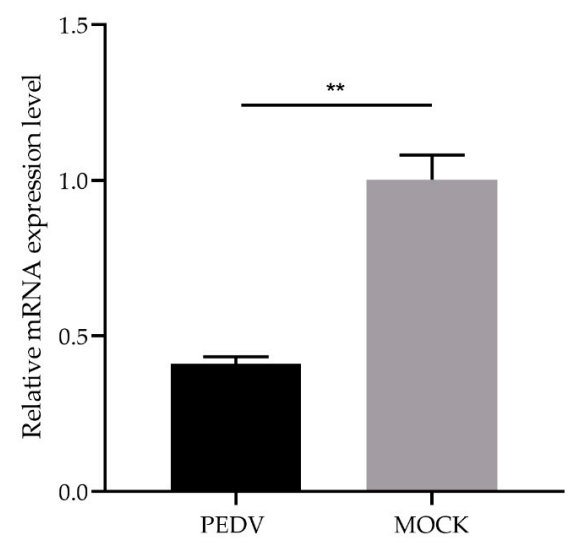

Figure 3. Expression changes of the GLP-2 gene in PEDV-infected IPEC-J2 cells. ${ }^{* *} p<0.01$. Bars represent the mean $\pm \mathrm{SD}(n=3)$.

\subsection{Verification of GLP-2 Gene Interference Efficiency in IPEC-J2 Cells}

To investigate the potential role of GLP-2 gene in regulating PEDV-infection, we constructed GLP-2 gene-silencing cells. After transfection of the GLP-2 gene shRNAs into IPEC-J2 cells, the red fluorescent protein could be expressed, indicating that shRNAs had been successfully transfected into cells and stably expressed (Figure 4A). The qPCR assay revealed that the interference efficiency of shRNA1 and shRNA2 reached $61 \%$, which could be used for subsequent experiments (Figure 4B). The above results indicated that the GLP-2 gene silencing cells had been successfully constructed. 
A

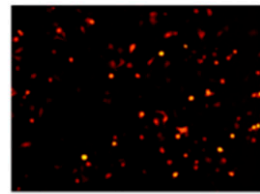

ShRNA NC

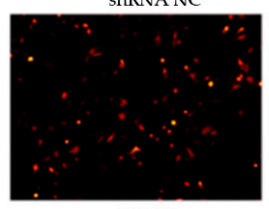

shRNA2

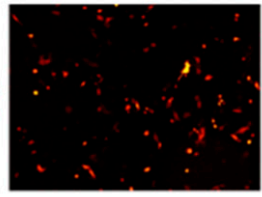

ShRNA1

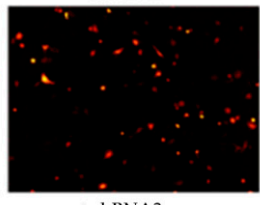

ShRNA3
B

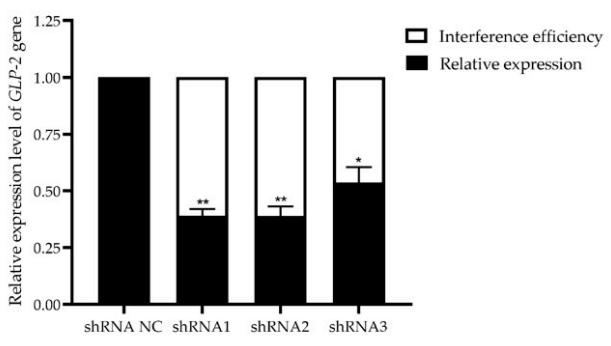

Figure 4. Verification of GLP-2 gene interference efficiency. (A) Transfection of GLP-2 gene shRNAs vector into IPEC-J2 cells. (B) GLP-2 gene mRNA expression level. shRNA NC represents negative control. shRNA1 shRNA2 and shRNA3 represent three RNA interference fragments of the GLP-2 gene. ${ }^{*} p<0.05,{ }^{* *} p<0.01$. Bars represent the mean $\pm \operatorname{SD}(n=3)$.

\subsection{Effect of GLP-2 Gene Silencing on PEDV Infection}

To further verify the effects of GLP-2 gene expression on PEDV infection, total RNA from shRNA1 and shRNA2 interfering cells infected by PEDV was extracted for M gene copy number detection. The results showed that the copy number of the PEDV M gene in the GLP-2 interfering cells was significantly higher than that in the control group $(p<0.01)$ (Figure 5A). Western blot analysis further validated the increased PEDV N protein expression in the GLP-2 interfering cells (Figure 5B). The results show that when the GLP-2 gene expression was down-regulated, the PEDV copy number was increased, indicating that GLP-2 gene may be involved in regulating the proliferation of PEDV in host cells.

A

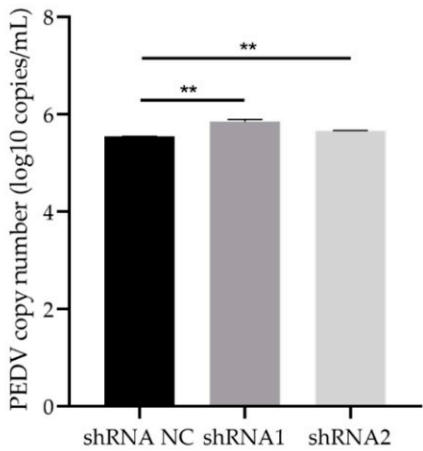

B

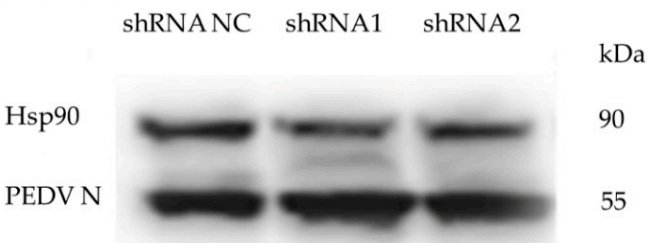

Figure 5. PEDV infects GLP-2 gene silent cell lines. (A) qPCR for determination of PEDV copy number in shRNA NC, shRNA1 and shRNA2 cells. (B) Western blot analysis of PEDV N protein. shRNA NC represents negative control. shRNA1 shRNA2 and shRNA3 represent three RNA interference fragments of GLP-2 gene. ${ }^{* *} p<0.01$. Bars represent the mean $\pm \operatorname{SD}(n=3)$.

\section{Discussion}

Piglet diarrhea is caused by many factors, of which PEDV-induced porcine epidemic diarrhea is the most common viral diarrhea disease in piglets, causing serious economic losses to the pig industry [23]. The small intestine is an important immune organ that resists the invasion of foreign pathogens through the intestinal epithelial villi. Therefore, maintaining the integrity of the intestinal mucosa is essential for animal health. We observed a damaged intestinal mucosa integrity induced by PEDV infection. It has been reported that GLP-2 is an intestinal protective factor that widely participates in the repair of intestinal mucosa [24]. Our study found that the GLP-2 gene is expressed in all intestinal segments, indicating that the GLP-2 gene may hae physiological functions in the intestinal mucosal immune responses. Expression of the GLP-2 gene in the jejunum and ileum of the intestinal mucosal damaged group was lower than that of the control group, indicating that 
PEDV infection may affect the integrity of intestinal mucosa by regulating the expression of the GPL-2 gene.

Studies have shown that GLP-2 receptor (GLP-2R) produces a large amount of cAMP by activating the activity of adenylate cyclase. GLP-2 can accelerate the differentiation, growth and repair of the entire intestinal crypt cell population through the cAMP-dependent signal transduction pathway mediated by GLP-2R [25]. GLP-2 can also promote the recovery of pathological intestinal mucosa and induce the proliferation of intestinal epithelial cells through the cell signal transduction pathway of mitogen-activated protein kinases [26]. Moreover, GLP-2 regulates the gene expression of small intestinal tight junction proteins. Tight junction proteins are an important connection method of intestinal epithelial cells and play an important role in maintaining the integrity of the intestinal barrier. After PEDV infection, the expression levels of tight junction proteins such as ZO-1, ZO-2, Occludin and Claudin-4 in the intestines significantly changed [22]. In addition, GLP-2 can stimulate the proliferation of small intestinal fossa cells to inhibit cell apoptosis, compensate for intestinal development disorders, and reduce enteritis symptoms in newborn animals $[27,28]$. We further detected the changes in GLP-2 gene expression at the cellular level and found expression of GLP-2 gene at $48 \mathrm{~h}$ after PEDV infection was significantly decreased, indicating that GLP-2 gene may be involved in the repair of intestinal mucosal damage induced by PEDV infection.

The most common way for a virus to infect the host is to destroy the epithelial barrier of the host cell, and tight junctions are crucial to maintaining the cell barrier [29]. Jung et al. [30] proved that PEDV infection caused changes in the structure of tight junction proteins ZO-1 and E-cadherin in the villi epithelium in vivo. Other viruses that cause diarrhea, such as rotavirus, can infect intestinal epithelial cells and the distribution of tight junction proteins Claudin-1 and Occludin, thereby promoting viral infections [31]. To further confirm the relationship between GLP-2 gene and PEDV, we silenced the expression of GLP-2 gene and observed the increased PEDV copies in host cells upon PEDV infection, which provided further supports for the participation of the GLP-2 gene in mediating the interactions between PEDV and host cells. The mechanisms of interaction between the GLP-2 gene and PEDV infection warrant further investigations.

\section{Conclusions}

In conclusion, we found that PEDV infection causes obvious pathological changes in the porcine intestines and that GLP-2 gene is involved in regulating PEDV infection. Furthermore, higher expression of GLP-2 gene may be beneficial to resist PEDV infection. Our findings provide novel insights into the functions of GLP-2 gene in response to PEDV infection and may contribute to the identification of resistant genes or genetic markers for disease resistance breeding in pigs.

Supplementary Materials: The following are available online at https://www.mdpi.com/2076-261 5/11/3/644/s1: Figure S1: Gel electrophoresis of tissue total RNA, Figure S2: qPCR amplification curves, Figure S3: qPCR melting curves.

Author Contributions: Conceptualization, W.B.; methodology, Y.Z.; software, Y.Z. and H.W.; formal analysis, Y.Z. and Z.R.; data curation, Y.Z.; validation, Y.Z. and H.W.; supervision, W.B. and S.W.; writing—original draft, Y.Z.; writing—review and editing, H.W., S.Z. and W.B. All authors have read and agreed to the published version of the manuscript.

Funding: This research was funded by the National Natural Science Foundation of China (31972535), Key Research and Development Project (Modern Agriculture) of Jiangsu Province (BE2019344, BE2019341) and the Priority Academic Program Development of Jiangsu Higher Education Institutions.

Institutional Review Board Statement: The study was conducted according to the Institutional Animal Care and Use Committee (IACUC) of the Yangzhou Univer-sity Animal Experiments Ethics Committee (permit number: SYXK (Su) IACUC 2012-0029). 
Data Availability Statement: The data presented in this study are available on reasonable request from the corresponding author.

Conflicts of Interest: The author declares that there is no potential conflict of interest.

\section{References}

1. King, A.M.Q.; Adams, M.J.; Carstens, E.B.; Lefkowitz, E.J. Virus Taxonomy: Ninth Report of the International Committee on Taxonomy of Viruses; Elsevier: Saint Louisl, MO, USA, 2011.

2. Kocherhans, R.; Bridgen, A.; Ackermann, M.; Tobler, K. Completion of the porcine epidemic diarrhoea coronavirus (PEDV) genome sequence. Virus Genes 2001, 23, 137-144. [CrossRef]

3. Duarte, M.; Gelfi, J.; Lambert, P.; Rasschaert, D.; Laude, H. Genome organization of porcine epidemic diarrhoea virus. Adv. Exp. Med. Biol. 1993, 342, 55-60. [PubMed]

4. Wang, K.; Lu, W.; Chen, J.F.; Xie, S.Q.; Shi, H.Y.; Hsu, H.J.; Yu, W.J.; Xu, K.; Bian, C.; Fisher, W.B.; et al. PEDV ORF3 encodes an ion channel protein and regulates virus production. FEBS Lett. 2012, 586, 384-391. [CrossRef] [PubMed]

5. Bosch, B.J.; Zee, R.V.D.; De Haan, C.A.M.D.; Rottier, P.J.M. The coronavirus spike protein is a class I virus fufsion protein: Structural and functional characterization of the fusion core complex. J. Virol. 2003, 77, 8801-8811. [CrossRef]

6. Klumperman, J.; Locker, J.K.; Meijer, A.; Horzinek, M.C.; Geuze, H.J.; Rottier, P.J. Coronavirus M proteins accumulate in the Golgi complex beyond the site of virion budding. J. Virol. 1994, 68, 6523-6534. [CrossRef] [PubMed]

7. Vennema, H.; Godeke, G.J.; Rossen, J.W.; Voorhout, W.F.; Horzinek, M.C.; Opstelten, D.J.; Rottier, P.J. Nucleocapsid-independent assembly of coronavirus-1ike particles by co-expression of viral envelope protein genes. EMBO J. 1996, 15, 2020-2028. [CrossRef]

8. Pensaert, M.B.; Debouck, P. A new coronavirus-like particle associated with diarrhea in swine. Arch. Virol. 1978, 58, 243-247. [CrossRef] [PubMed]

9. Molloy, M.; Bouladoux, N.; Belkaid, Y. Intestinal Microbiota: Shaping local and systemic immune responses. Semin. Immunol. 2012, 24, 58-66. [CrossRef] [PubMed]

10. Blikslager, A.T.; Moeser, A.J.; Gookin, J.L.; Jones, S.L.; Odle, J. Restoration of barrier function in injured intestinal mucosa. Physiol. Rev. 2007, 87, 545-564. [CrossRef]

11. Laukoetter, M.G.; Bruewer, M.; Nusrat, A. Regulation of the intestinal epithelial barrier by the apical junctional complex. Curr. Opin. Gastroenterol. 2006, 22, 85-89. [CrossRef]

12. Tsukita, S.; Furuse, M.; Itoh, M. Multifunctional strands in tight junctions. Nat. Rev. Mol. Cell Biol. 2001, 2, 285-293. [CrossRef]

13. Zhao, Y.; Qin, G.X.; Sun, Z.W.; Che, D.S.; Bao, N.; Zhang, X.D. Effects of soybean agglutinin on intestinal barrier permeability and tight junction protein expression in weaned piglets. Int. J. Mol. Sci. 2011, 12, 8502-8512. [CrossRef]

14. Mowat, A.M. Anatomical basis of tolerance and immunity to intestinal antigens. Nat. Rev. Immunol. 2003, 3, 331-341. [CrossRef]

15. Drucker, D.J. Biological actions and therapeutic potential of the glucagon-like peptides. Gastroenterology 2002, 122, 531-544. [CrossRef]

16. Heuvel, E.D.; Wallace, L.; Sharkey, K.A.; Sigalet, D.L. Glucagon-like peptide 2 induces vasoactive intestinal polypeptide expression in enteric neurons via phosphatidylinositol 3-kinase- $\gamma$ signaling. Am. J. Physiol. Endocrinol. Metab. 2012, 303, E994-E1005. [CrossRef]

17. Brubaker, P.L.; Anini, Y. Direct and indirect mechanisms regulating secretion of glucagon-like peptide-1 and glucagon-like peptide-2. Can. J. Physiol. Pharm. 2003, 81, 1005-1012. [CrossRef]

18. Drucker, D.J.; Erlich, P.; Asa, S.L.; Brubaker, P.L. Induction of intestinal epithelial proliferation by glucagon-like peptide 2. Proc. Natl. Acad. Sci. USA 1996, 93, 7911-7916. [CrossRef]

19. Petersen, Y.M.; Hartmann, B.; Holst, J.J.; Huerou-Luron, I.L.; Bjørnvad, C.R.; Sangild, P.T. Introduction of enteral food increases plasma GLP-2 and decreases GLP-2 receptor mRNA abundance during pig development. J. Nutr. 2003, 133, 1781-1786. [CrossRef] [PubMed]

20. Benjamin, M.A.; Mckay, D.M.; Yang, P.C.; Cameron, H.; Perdue, M.H. Glucagon-like peptide-2 enhances intestinal epithelial barrier function of both transcellular and paracellular pathways in the mouse. Gut 2000, 47, 112-119. [CrossRef]

21. Moran, J.W.; O'neill, C.; Mclaughlin, J.T. GLP-2 enhances barrier formation and attenuates TNF $\alpha$-induced changes in a Caco-2 cell model of the intestinal barrier. Regul. Pept. 2012, 178, 95-101. [CrossRef] [PubMed]

22. Zong, Q.F.; Huang, Y.J.; Wu, L.S.; Wu, Z.C.; Wu, S.L.; Bao, W.B. Effects of porcine epidemic diarrhea virus infection on tight junction protein gene expression and morphology of the intestinal mucosa in pigs. Pol. J. Vet. Sci. 2019, 211, 345-353.

23. Jung, K.; Wang, Q.; Scheuer, K.A.; Lu, Z.; Zhang, Y.; Saif, L.J. Pathology of US porcine epidemic diarrhea virus strain PC21A in gnotobiotic pigs. Emerg. Infect. Dis. 2014, 20, 662-665. [CrossRef]

24. Yusta, B.; Somwar, R.; Wang, F.; Munroe, D.; Grinstein, S.; Klip, A.; Drucker, D.J. Identification of glucagon-like peptide-2(GLP2)-activited signaling pathways in baby hamster kidney fibroblasts expressing the rat GLP-2 receptor. J. Biol. Chem. 1999, 274, 30459-30467. [CrossRef]

25. Jasleen, J.; Shimoda, N.; Shen, E.R.; Tavakkolizaden, A.; Whang, E.E.; Jacobs, D.O.; Zinner, M.J.; Ashley, S.W. Signaling mechanisms of glucagon-like peptide 2-induced intestinal epithelial cell proliferation. J. Surg. Res. 2000, 90, 13-18. [CrossRef]

26. Cameron, H.L.; Perdue, M.H. Stress impairs murine intestinal barrier function: Improvement by glucagon-like peptide-2. J. Pharm. Exp. 2005, 313, 214-220. [CrossRef] [PubMed] 
27. Cameron, H.L.; Yang, P.C.; Perdue, M.H. Glucagon-like peptide-2 enhanced barrier function reduces pathophysiology in a model of food allergy. Am. J. Physiol. Gastrointestiest. Liver Physiol. 2003, 284, G905-G912. [CrossRef]

28. Boushey, R.P.; Yusta, B.; Druckey, D.J. Glucagon-like peptide (GLP)-2 reduces chemotherapy-associated mortality and enhances cell survival in cells expressing a transfected GLP-2 receptor. Cancer Res. 2001, 61, 687-693. [PubMed]

29. Jinguji, Y.; Ishikawa, H. Electron microscopic observations on the maintenance of the tight junction during cell division in the epithelium of the mouse small intestine. Cell Struct. Funct. 1992, 17, 27-37. [CrossRef]

30. Jung, K.; Eyerly, B.; Annamalai, T.; Lu, Z.Y.; Saif, L.J. Structural alteration of tight and adherens junctions in villous and crypt epithelium of the small and large intestine of conventional nursing piglets infected with porcine epidemic diarrhea virus. Vet. Microbiol. 2015, 177, 373-378. [CrossRef]

31. Dickman, K.G.; Hempson, S.J.; Anderson, J.; Lippe, S.; Zhao, L.; Burakoff, R.; Shaw, R.D. Rotavirus alters paracellular permeability and energy metabolism in Caco-2 cells. Am. J. Physiol. Gastrointestiest. Liver Physiol. 2000, 279, G757-G766. [CrossRef] [PubMed] 